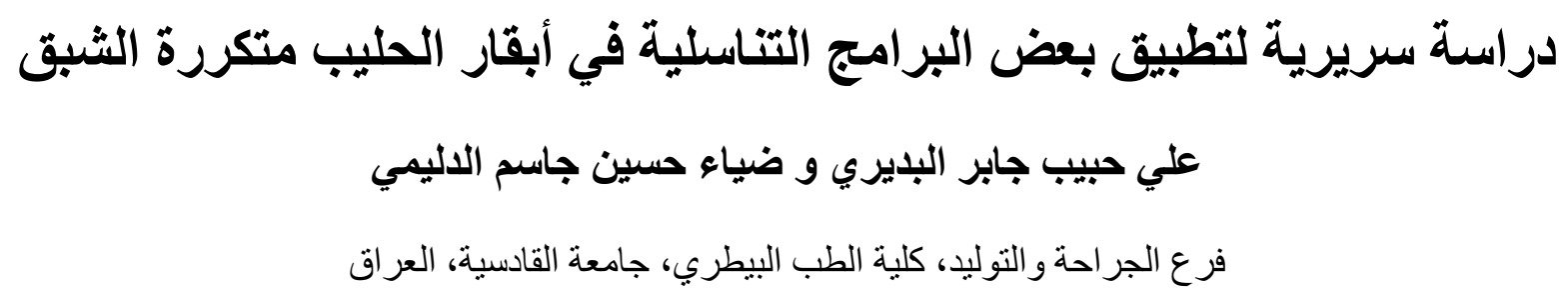

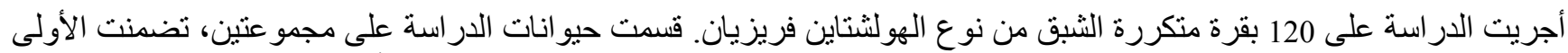
60 (A)

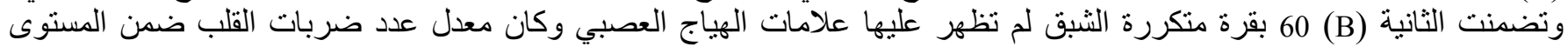

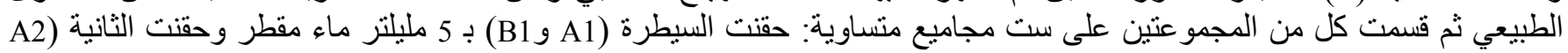

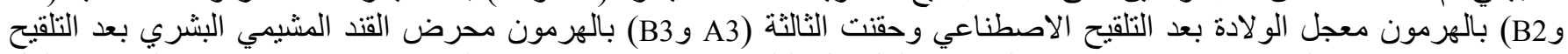

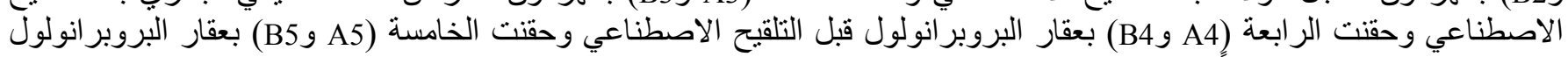

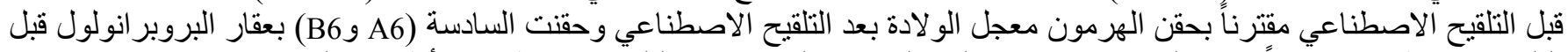

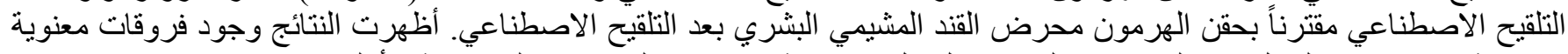

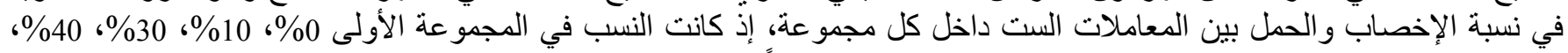

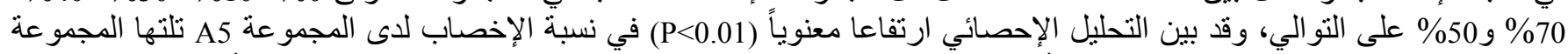

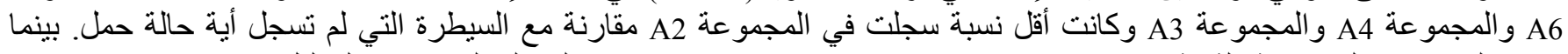
كانت النسب في المجموعة الثانية 10\% و

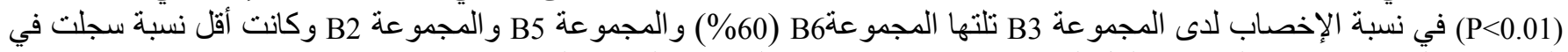

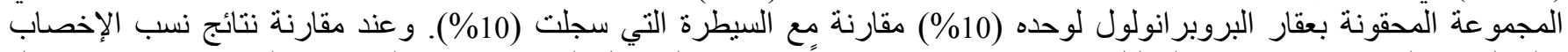

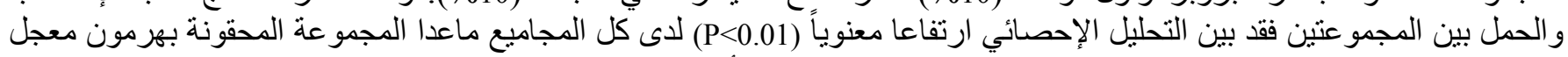

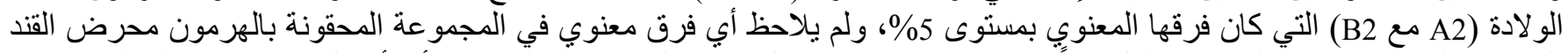

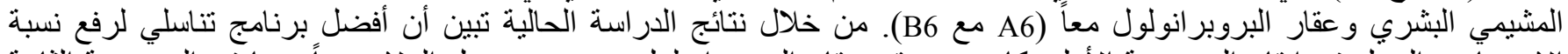

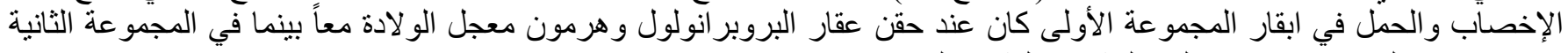

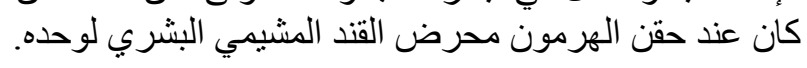

\title{
A clinical study for use of some reproductive programs in repeat-breeder dairy cows
}

\author{
A.H.J. Al-Bdeery and D.H.J. Al-Delemy \\ Department of Surgery and Obstetrics, College of Veterinary Medicine, Al-Qadisiya University, Iraq
}

\section{Abstract}

The present study was carried out on 120 repeat-breeder dairy cows belong to the breed Holstien-Frriesian, Animals of the study was divided into two equal groups, first group (A) involved 60 repeat breeder dairy cows expressed nervous excitation signs and elevated heart rate at the time of artificial insemination, while second group (B) involved 60 repeat-breeder dairy cows did not express nervous excitation signs and have normal heart rate. Each group was further subdivided into six equal subgroups: A1 and B1 (control) was injected with $5 \mathrm{ml}$ of distilled water, A2 and B2 was injected with Oxytocin after artificial insemination, A3 and B3 was injected with hCG after AI, A4 and B4 was injected with Propranolol before AI, A5 and B5 was injected with Propranolol before AI in addition to injection of Oxytocin after AI, A6 and B6 was injected with Propranolol before $\mathrm{AI}$ in addition to injection of hCG after AI. The result revealed significant differences of the conception and pregnancy rate among the sixth subgroups inside each group. The percentage of repeat-breeder dairy cows belonging A1, A2, A3, A4, A5 


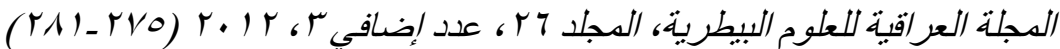 وقائع الهؤتهر العلمي السادم، كلية الطب البيطري، جامعة الدوصل}

and $\mathrm{A} 6$ were $0 \%, 10 \%, 30 \%, 40 \%, 70 \%$ and $50 \%$, respectively. The statistical analysis showed significant increase $(\mathrm{P}<0.01)$ in subgroup (A5) (70\%) followed by A6 (50\%), A4 (40\%), A3 (30\%) and the lowest ratio was recorded in A2 (10\%) compared with control (A1) which did not record any instance of pregnancy. While the ratios of the repeat-breeder dairy cows subgroups which did not expressed nervous excitation (B1, B2, B3, B4, B5 and B6) were $10 \%, 20 \%, 60 \%, 10 \%, 20 \%$ and $60 \%$, respectively. The statistical analysis revealed significant differences $(\mathrm{P}<0.01)$ in the conception rate of $\mathrm{B} 6(60 \%)$ followed by B3 (60\%), B2 and B5 (20\%) while the lowest ratio was recorded in B4 (10\%) and (control) $(10 \%)$. On the other hand, in comparison between the groups (A and B), the results revealed higher ratios of all subgroups belong to A group at 0.01 level of significancy except A2 and B2 which revealed difference at 0.05 level of significancy, while A6 and B6 showed no significant difference. The result of present study stated that the better reproductive program to increase the conception and pregnancy rate in the first group A was by injection of Propranolol in addition to Oxytocin hormone, while second group B was by injection of hCG hormone only.

Available online at http://www.vetmedmosul.org/ijvs

تطبيق تللك البرامج للحفاظ على التناسل الناجح لهذه القطعان وزيادة كفاءتها الإنتاجية.

لذللك صممت هذه الدر اسة للوصول الإناجة إلى أفضل برنامج تناسلي

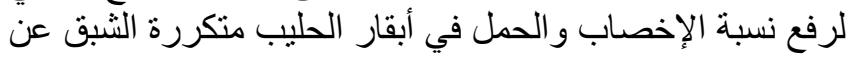
طريق استخدام عدد من الهرمونات وبطرق مختلفة.

\section{المواد وطر ائق العمل}

حيوانات الدراسة

أجريت الدراسة ألحالية للمدة من تشرين الثاني 2009 ولغاية

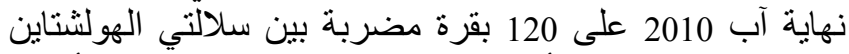

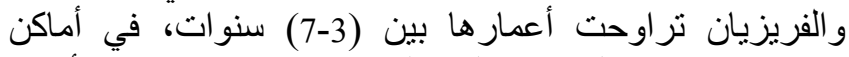

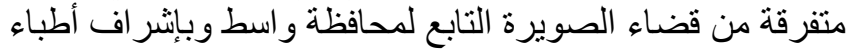

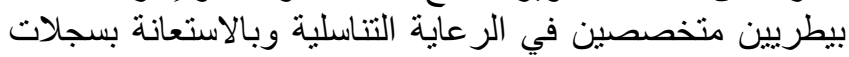

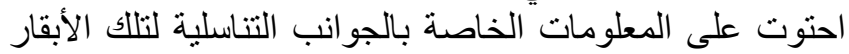

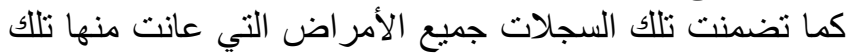

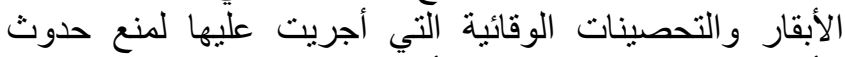

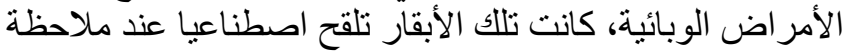

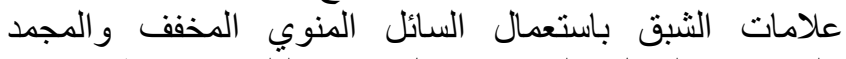

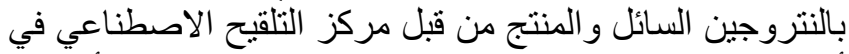

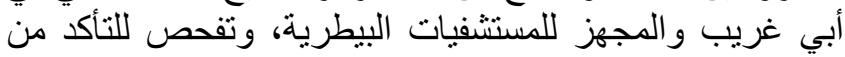
حذوث الحمل عن طريق الجس عبر المستقيم بعد شهرين من تلقيحها.

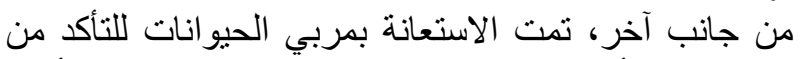

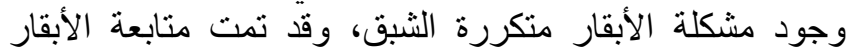

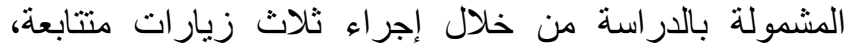

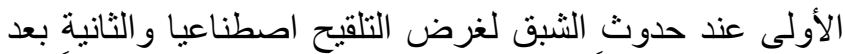

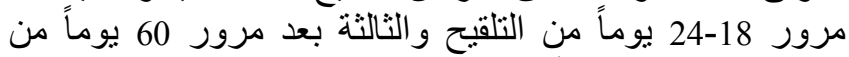

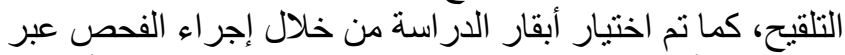

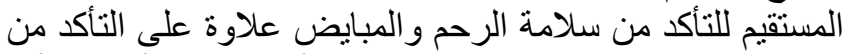

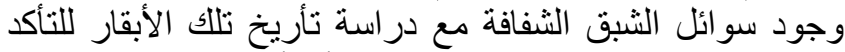

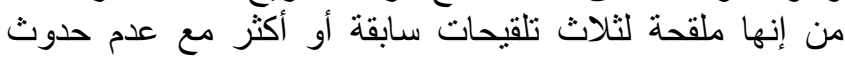

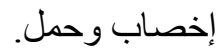

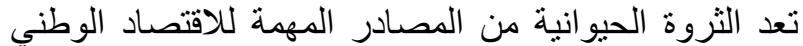

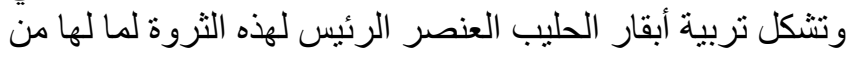

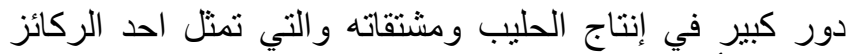

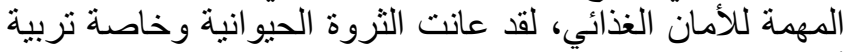

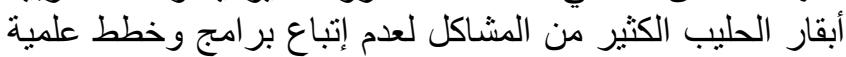

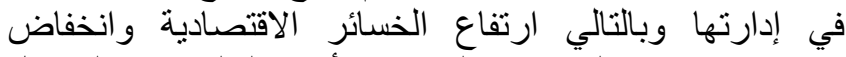

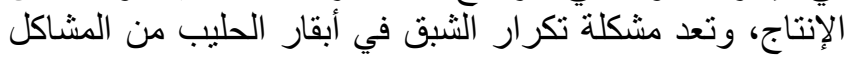

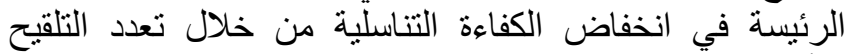

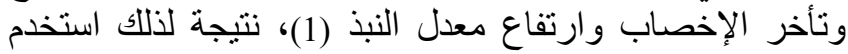
Sequin (hCG) human Chorionic Gonadotropin الإن الرفع (GnRH) Gonadotropin-Releasing Hormone الإخصاب والحمل في الأبقار متكررة الشبق، كما استخدم

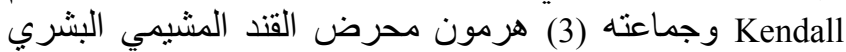

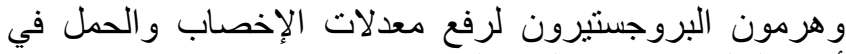

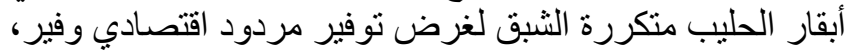

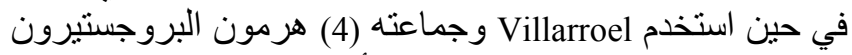

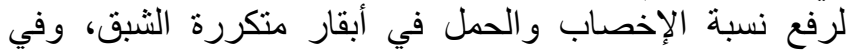

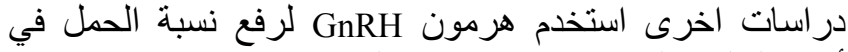

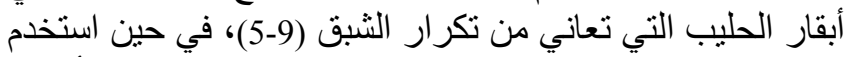

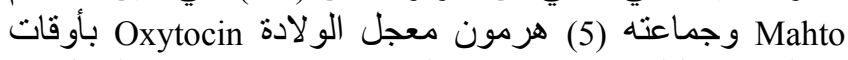

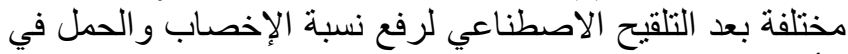

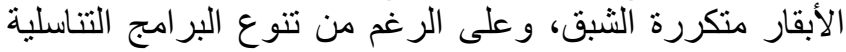

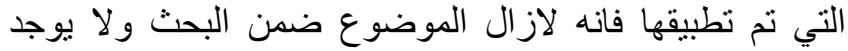

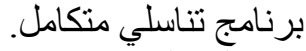

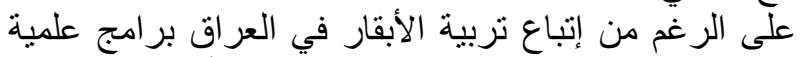

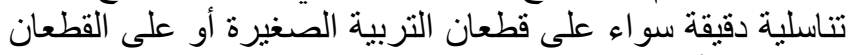

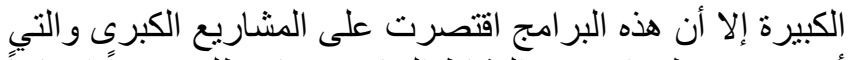

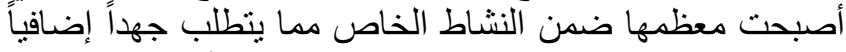

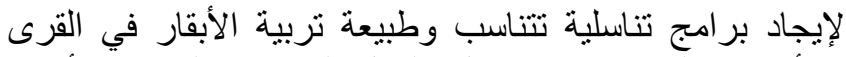

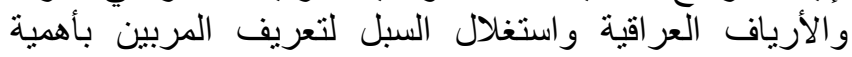




\section{نسبة الإخصاب و الحمل}

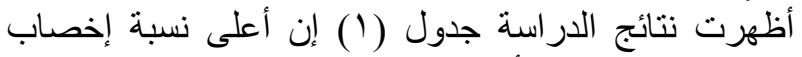

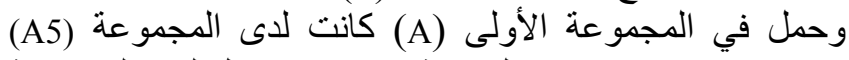

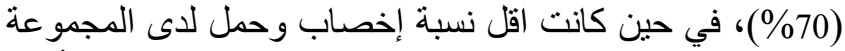

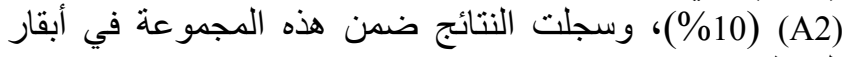

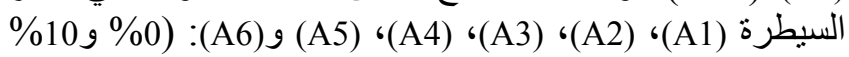

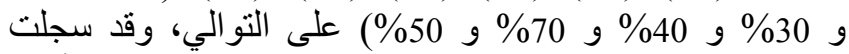

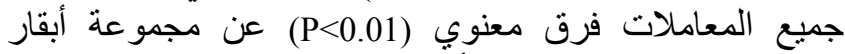

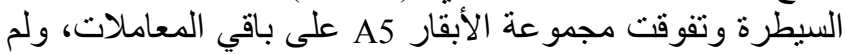

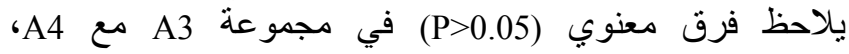

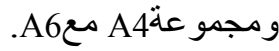

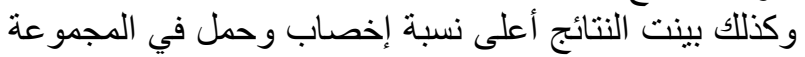

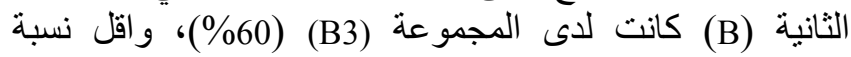

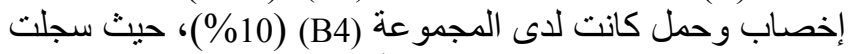

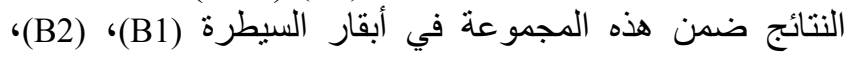

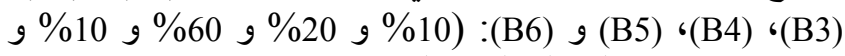

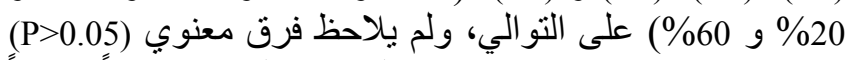

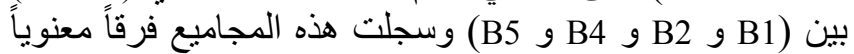

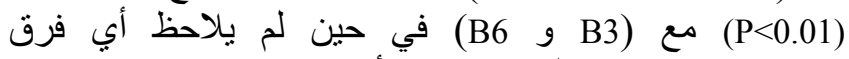
معنوي(P>0.05) بين المجمو عتين الأخيرتين. و وعند مقارنة النتائج إحصائيا لنفس الإيرتين المعاملة بين المجموعة

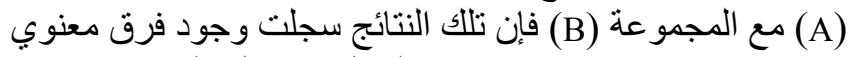

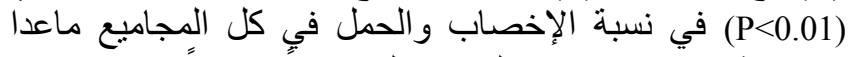

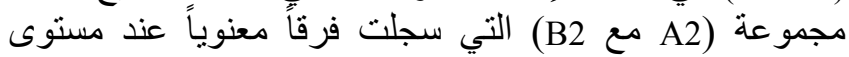

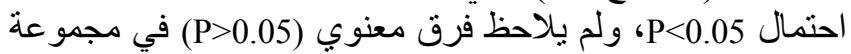

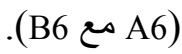

\section{معدل عدد ضربات القلب /الدقيقة في أبقار الحليب متكررة}

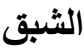

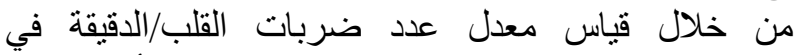

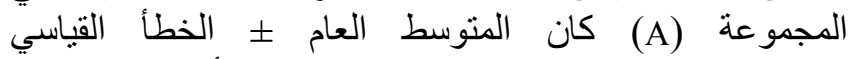

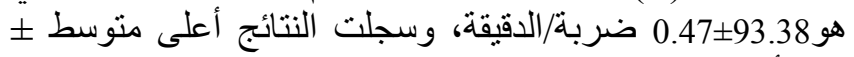
الخطأ القياسي لعدد ضربات القلب كان

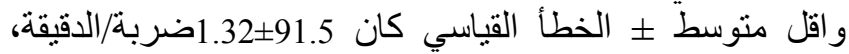

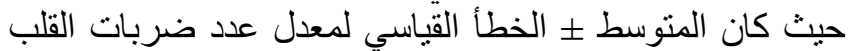

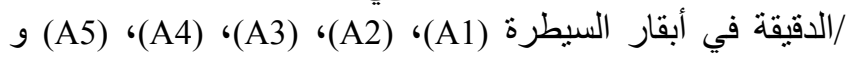

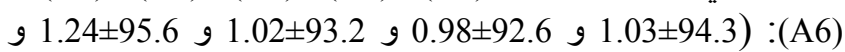

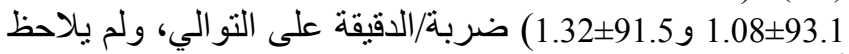
أي فرق معنوي (P>0.05) لمعدل عدد ضربات القلب /الدقيقة هذه

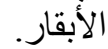

في حين المتوسط العام د الخطأ القياسي لمعدل عدد ضربات

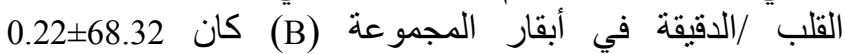

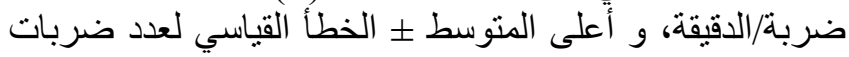

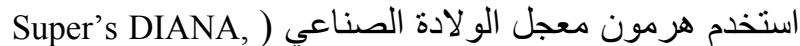

S.L., CTRA. C-17,Km17, 08150 Parets del Valles الو (Barcelona) ESPANA

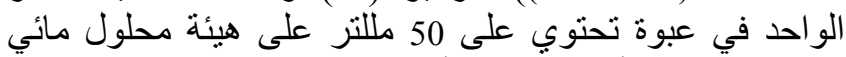

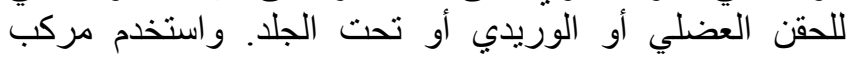

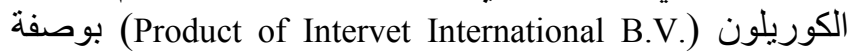
مصدر للهرمون محرض القند المشيمي البشري والذي يكون

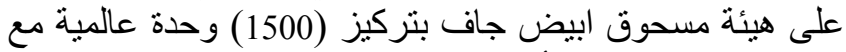
المذيب للحقن العضلي أو الوريدي. واستخدم عقار البروبر انولولئ عالون (Lab. RENAUDIN-6425 ITXASSOU-FRANCE) محلول مائي بتركيز 1 ملغر ام للملتر الواحد في عبوة تحتوي 1مل للحقن الوريدي البطيء.

تصميم الدراسة

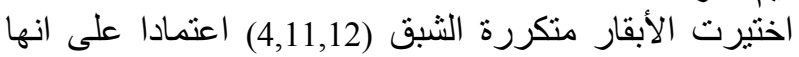

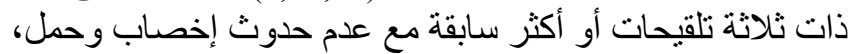

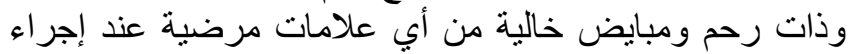
الجس ألمستقيمي، وذات سو ائل شبق التِ رائقة.

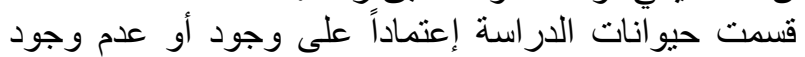

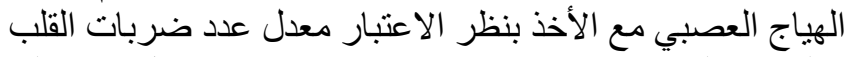

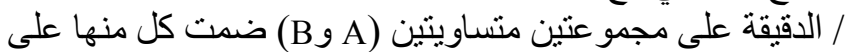

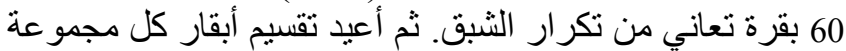

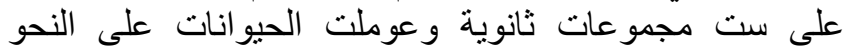

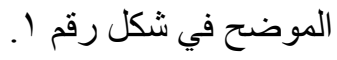

لقحت أبقار الأدراسة اصطناعيا باستعمال السائل المنوي

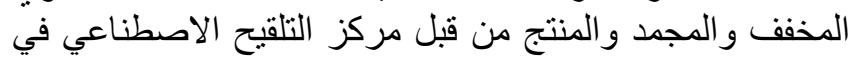

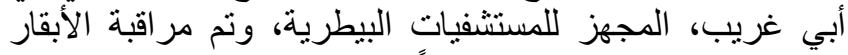

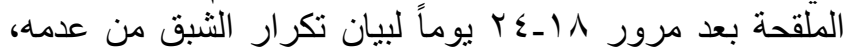

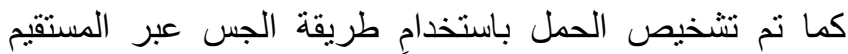

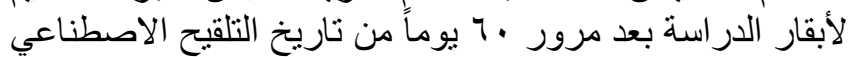

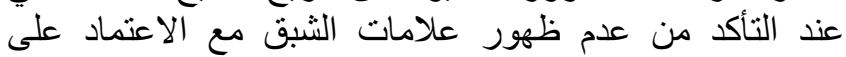

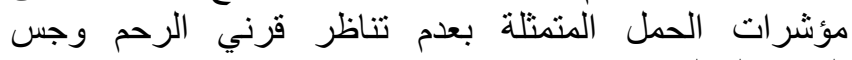
الحويصلة الجنينية.

التحليل الإحصائي حللت نتائج الدراسة إحصائيا و واستخدم المعدل والخطأ

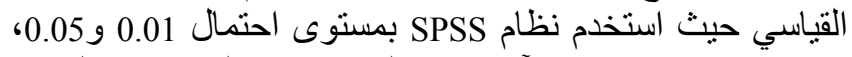

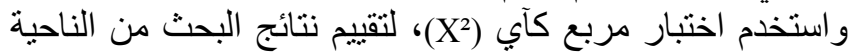

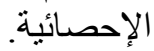




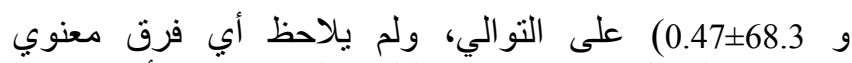

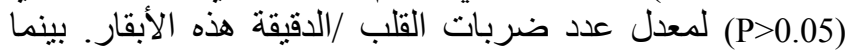

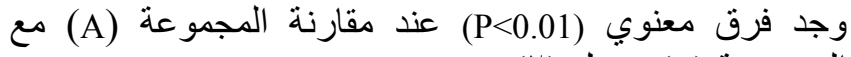

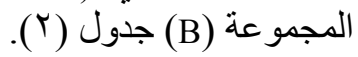

القلب ضمن هذه الأبقار كان 69.3 0.33 ضرببة/الدقيقة وأقل

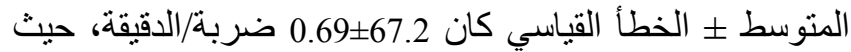

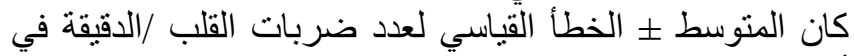

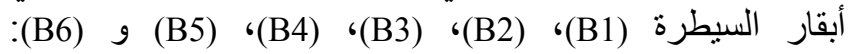
$0.41 \pm 68.1,0.42 \pm 690.33 \pm 69.3$ و $0.69 \pm 67.2$ و $0.58 \pm 68)$

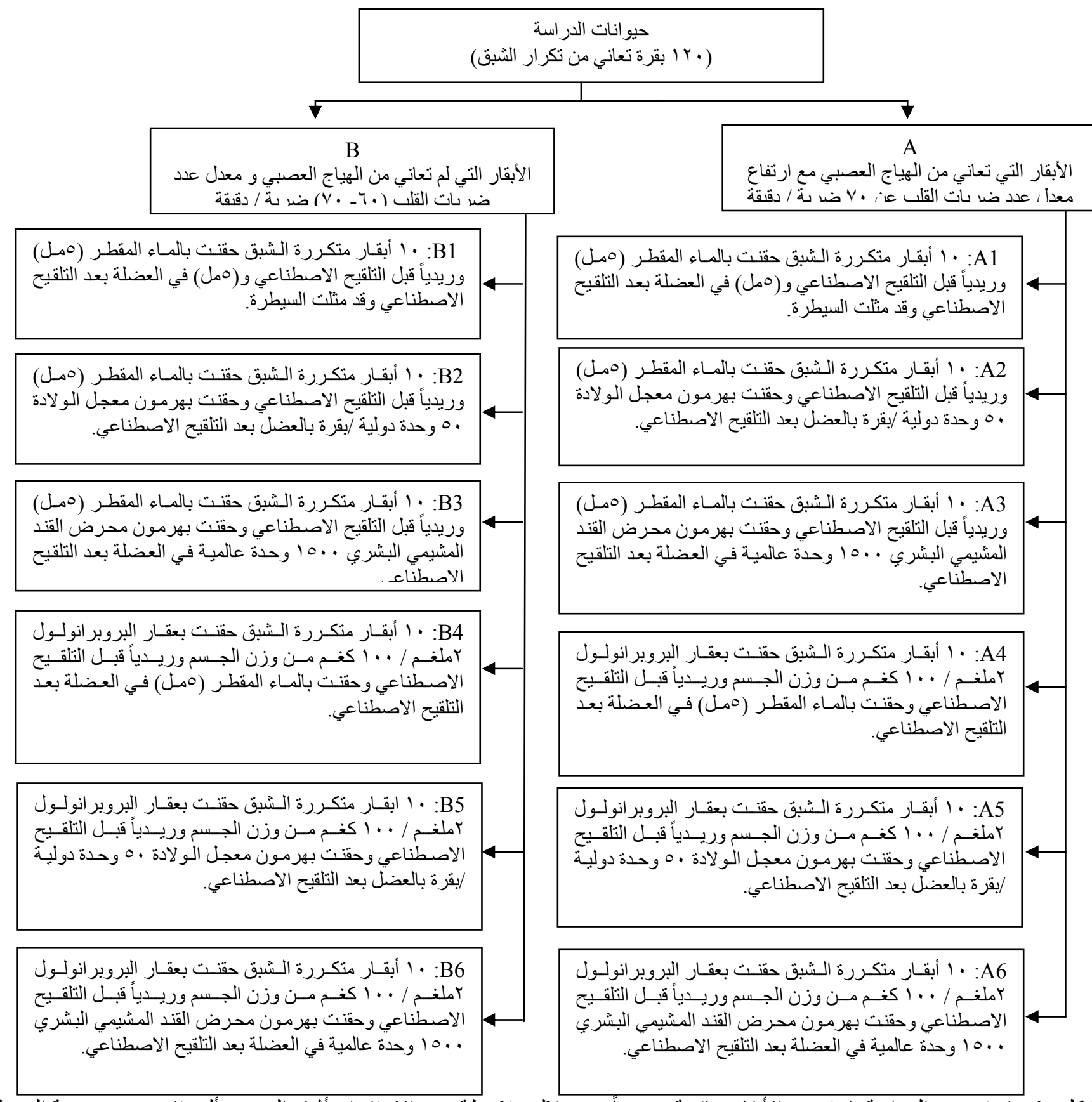

شكل رقم ا: تصميم الدر اسة. اعتبرت الأبقار هائجة عصبياً من خلال ملاحظة عدم الاستقر ار أثناء الفحص ألمستقيمي و صعوبة الفئة السيطرة

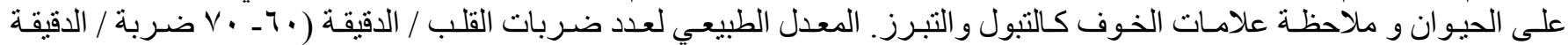




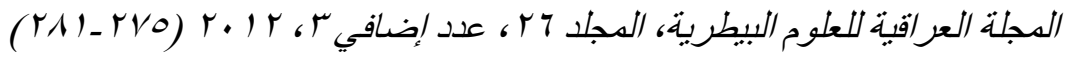

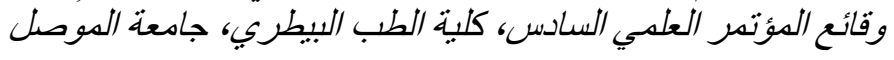

جدول (1): يمثل تأثير حقن هرمون معجل الولادة وهرمون محرض القند المشيمي البشري وعقار البروبر انولول على نسبة الإخصاب

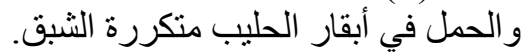

\begin{tabular}{|c|c|c|c|c|c|}
\hline \multicolumn{2}{|c|}{ غير الحامل } & \multicolumn{2}{|c|}{ الحو امل } & \multirow{2}{*}{ 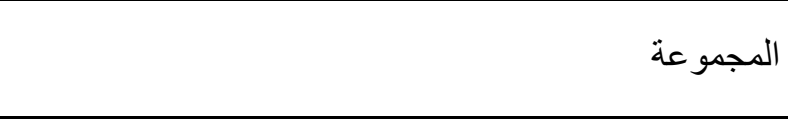 } & \multirow{2}{*}{ الأبقار الملقحة } \\
\hline النسبة \% & 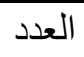 & النسبة \% & 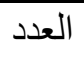 & & \\
\hline 100 & 10 & $0 * \mathrm{a}$ & -- & السيطرة (A1) & \\
\hline 90 & 9 & $10^{\bullet b}$ & 1 & المحقونة بهرمون معجل الو لادة & الأبقار ذات الهياج \\
\hline 70 & 7 & $30^{* \mathrm{c}}$ & 3 & المحقونة بهرمون محرض القند المشيمي البشري (A3) & العصبي وارتفاع \\
\hline 60 & 6 & $40^{* \mathrm{ce}}$ & 4 & المحقونة بعقار البروبر انولول & \\
\hline 30 & 3 & $70^{* d}$ & 7 & المحقونة بعقار البروبر انولول وهرمون معجل الو لادة & 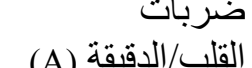 \\
\hline 50 & 5 & $50^{\mathrm{e}}$ & 5 & المشيمي البشري بعقار البروبر انولول و هرمون محرض القند & \\
\hline 90 & 9 & $10^{* \mathrm{a}}$ & 1 & (B1) السيطرة & الأبقار \\
\hline 80 & 8 & $20^{\bullet a}$ & 2 & (B2) المحقونة بهرمون معجل الو لادة & يلاحظ عليها مالم \\
\hline 40 & 4 & $60^{* b c}$ & 6 & (B3) المحقونة بهرمون محرض القند المشيمي البشري & علامات الهياج \\
\hline 90 & 9 & $10^{* a}$ & 1 & 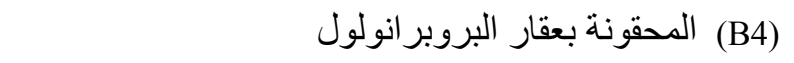 & العصبي أو \\
\hline 80 & 8 & 20 *a & 2 & (B5) المحقونة بعقار البروبر انولول وهرمون معجل الولادة & الارتفاع في معدل \\
\hline 40 & 4 & $60^{\mathrm{c}}$ & 6 & المشيمي البشري بحقة بعقار البروبر انولول و هرمون محرض القند & القلب/الدقيقة (B) \\
\hline
\end{tabular}

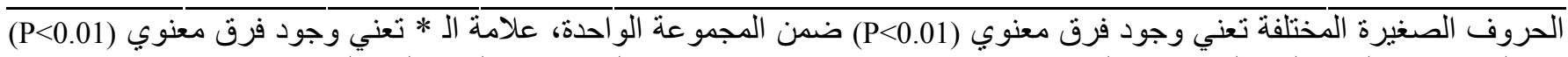

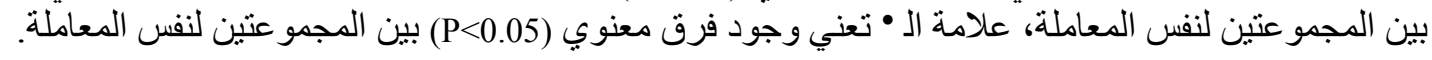

جدول(r) معدل عدد ضربات القلب /الدقيقة في أبقار الحليب متكررة الثبق

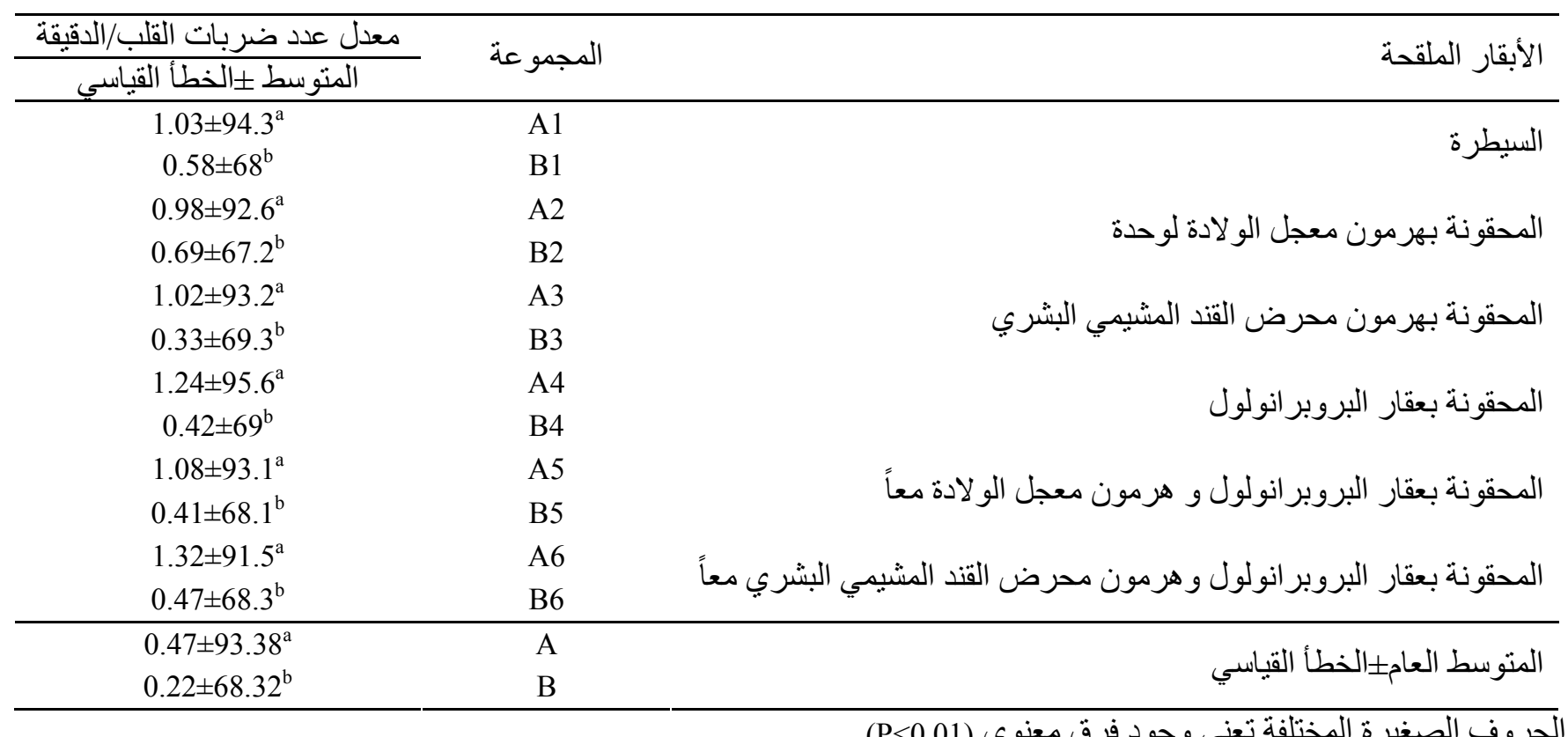

الحروف الصغيرة المختلفة تعني وجود فرق معنوي (P<0.01). 
المهاد حيث يقلل من تحرير GnRH والأخير يقلل من طرح

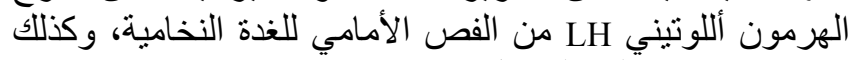

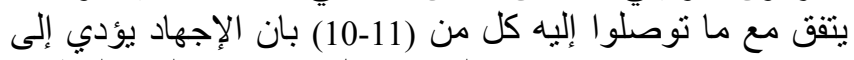

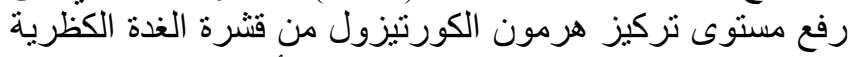

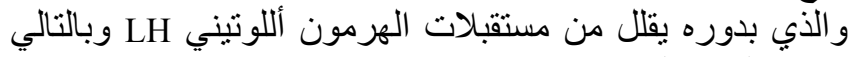

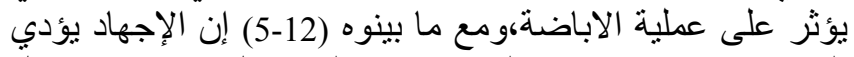

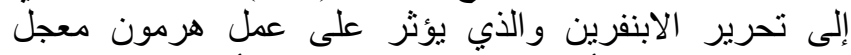

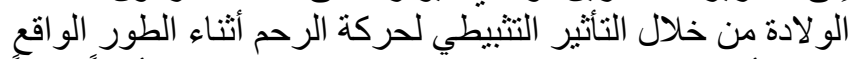

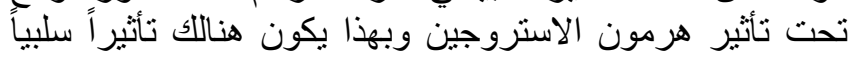

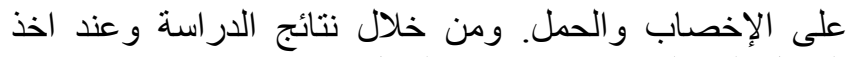

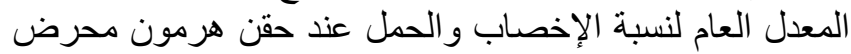

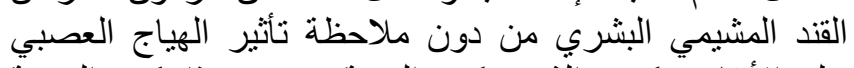

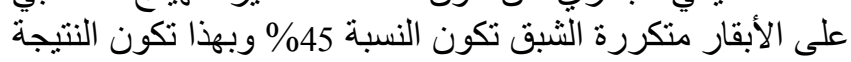

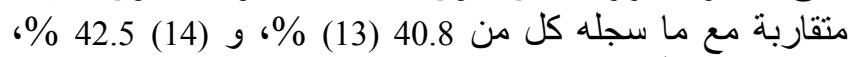

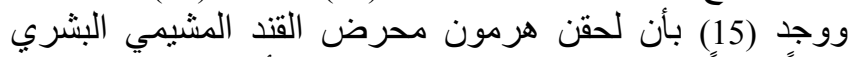

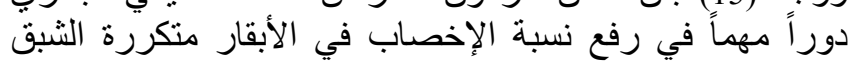

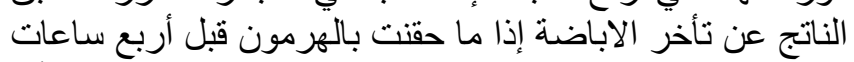

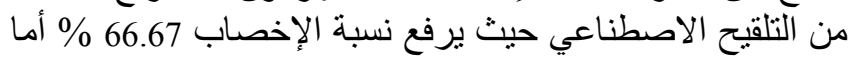

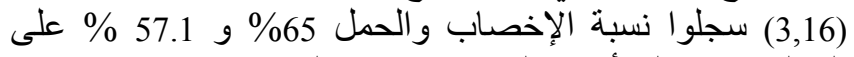
التو الي نتيجة إلى أهمية الوقت الإبل عند حقن الهرمون.

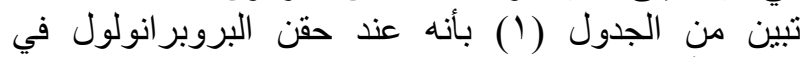

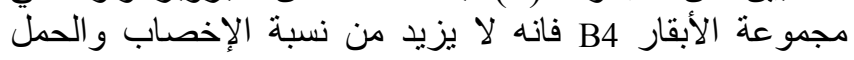

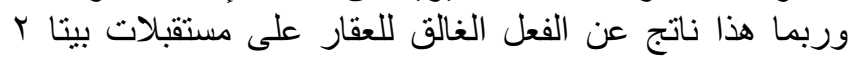

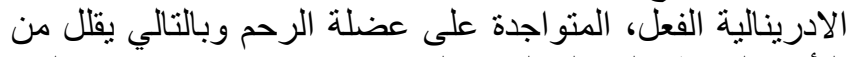

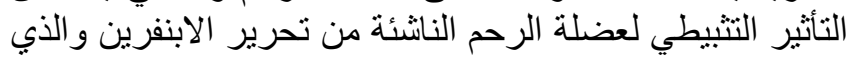

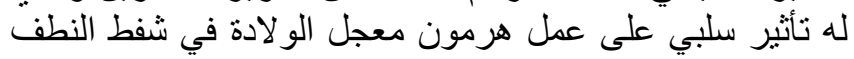

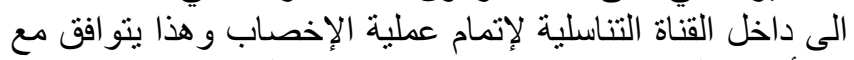

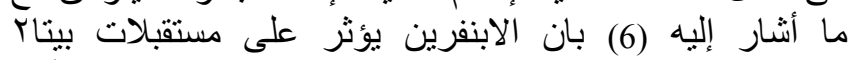

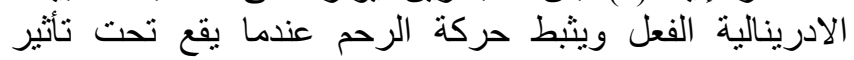

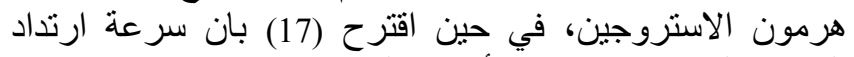

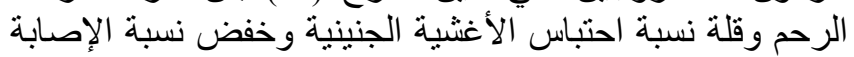

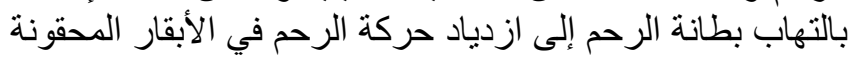

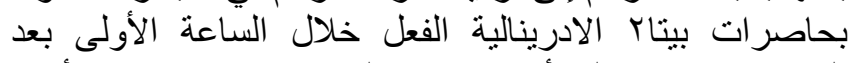
الولادة وهذا يكون له تأثير ايجابي على نسبة الإخصاب في أبقار

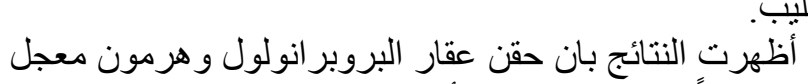

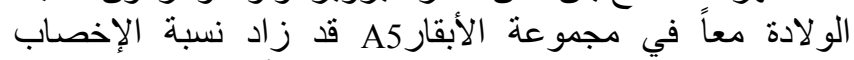

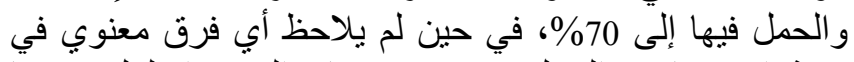

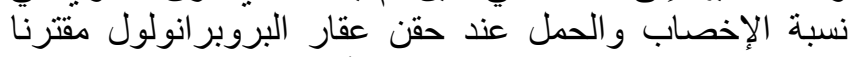

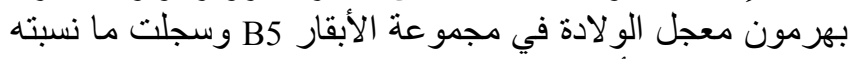

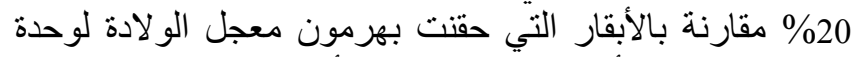

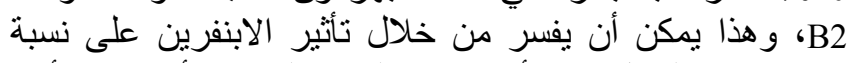

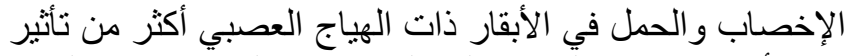

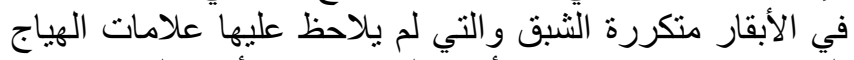
العصبي، و هذا يتفق مع ما أشار إليه (5) بان الأبقار المجهدة إنثاء
المناقشة

أظهرت نتائج الدر اسة وجود فرق معنوي (P<0.05) في نسبة

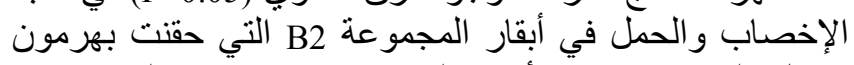

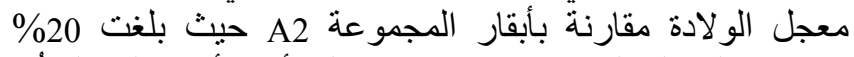

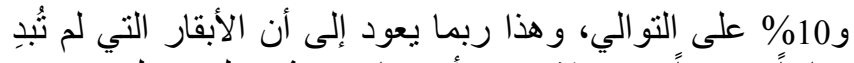

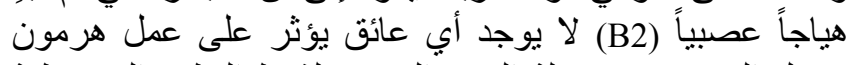

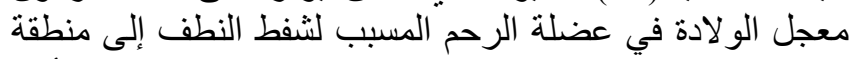

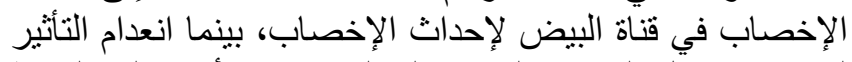

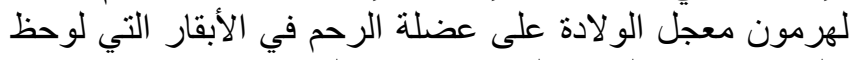

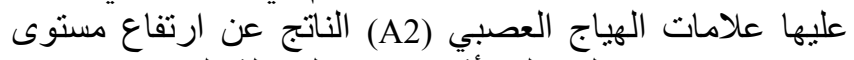

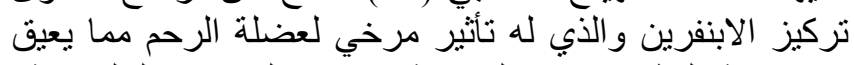

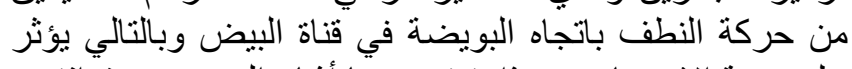

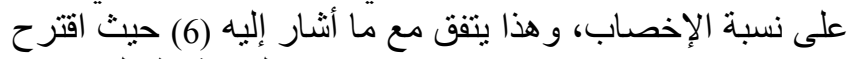

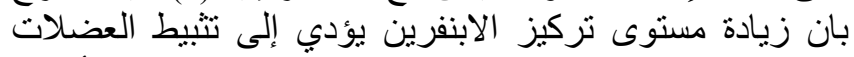

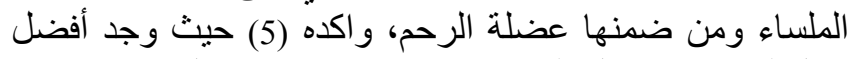

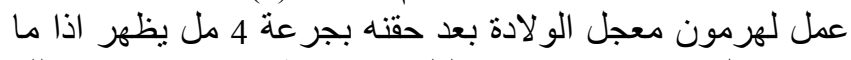

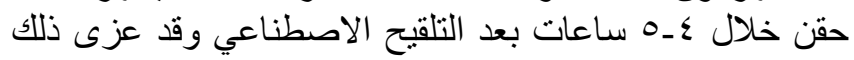

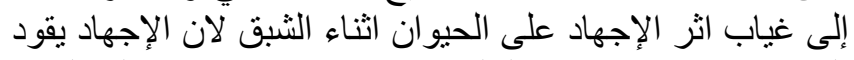

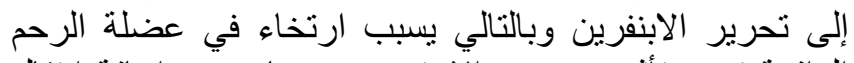

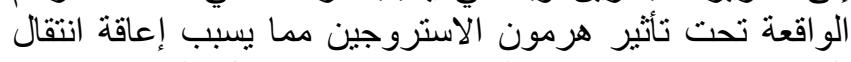

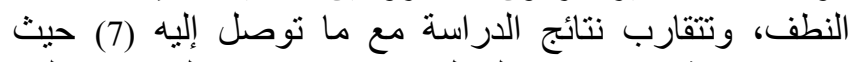

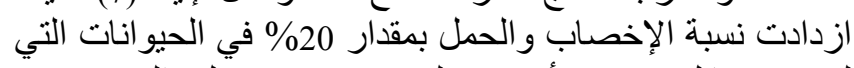

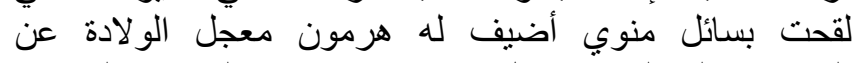

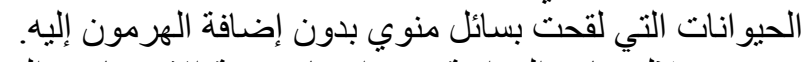

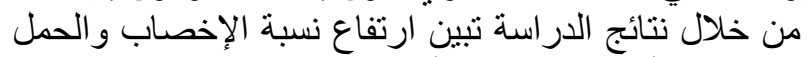

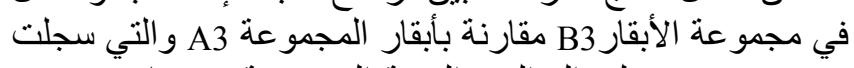

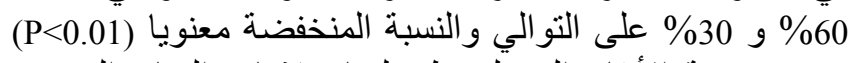

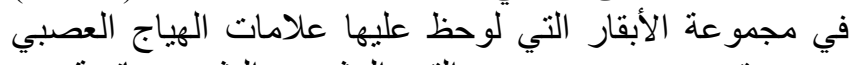

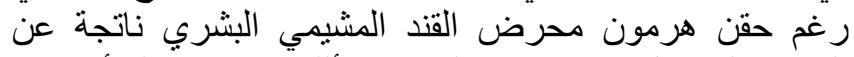

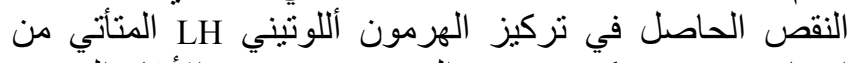
ارتفاع مستوى تركيز هرمون البركيز البروجستيرون في الأبقار المجهدة

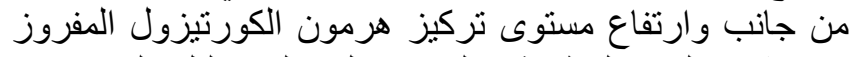

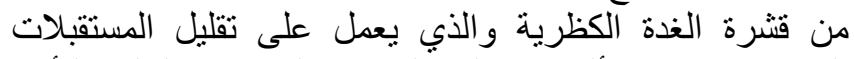

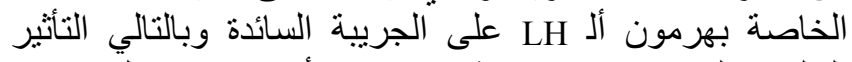

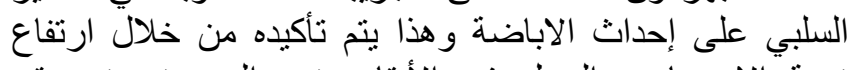

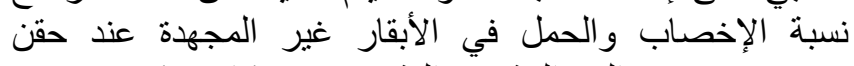

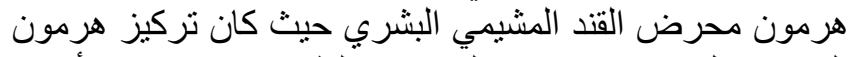

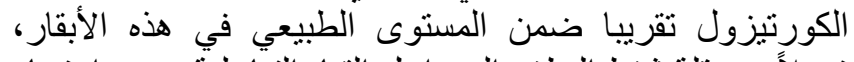

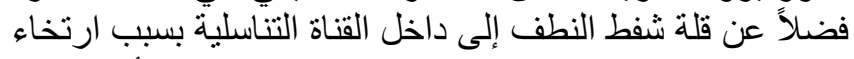

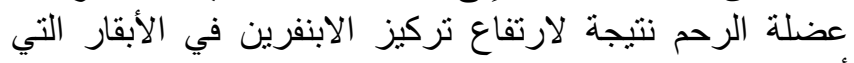

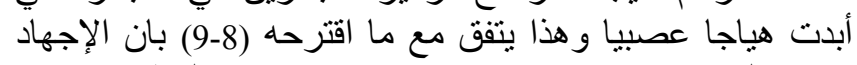

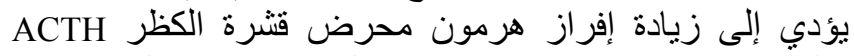

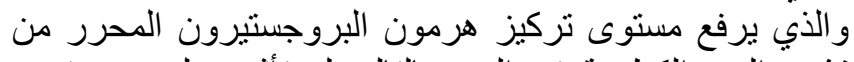

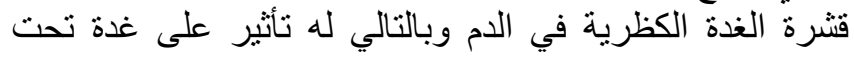




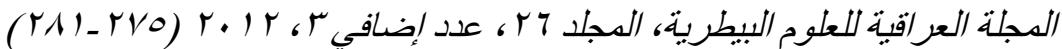

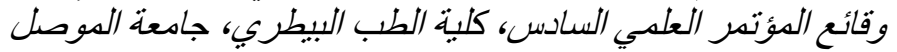

descriptive epidemiology \& estimated economic impact. Theriolog. , 26:309-322.

2. 2-Sequin, B. ; Cnvey, E. \& Oxender, W. (1976). Effect of gonadotropin- releasing hormone \& human chorionic gonadotropin on cows with ovarian follicular cysts. Ainm. J. Vet. Res., 73:153.

3. 3-Kendall, N.R. ; Flint, A.P. \& Mann, G.E. (2009). Incidence and treatment of inadequate postoulatory progesterone concentrations in repeat breeder cows.U.S. National Library. Vet. J., 181:158-162.

4. 4-Villarrorel, A. ; Muartino, A. ; BonDurant, R. H. ; Deletang, F. \& Sischo, M. (2004). Effect of post-insemination supplementation with PRID on pregnancy in repeat-breeder Holstein cows. Theriogenology ,61:1513-1520.

5. 5- Mahto, D. ; Singh, B. ; Adil, A. \& Verma, R.K. (2008). Effect of oxytocin \& cofecu on post-insemination conception rate in repeat breeder cattle on subsequent time interval. Vet. World ,Vol.1, 9:268269.

6. 6- Rastogi, S.G. (2007). Essential of animal physiology. $4^{\text {th }}$ edition, New Age , International, Ltd, Publishers, Ansari Road, Daryaganj, NewDelhi.Pp.419-425 \& 444-446.

7. 7- Gibson, S. ; Tempelan, R.J. \& Kirwood, R.N. (2004). Effect of oxytocin - supplemented semen on fertility of sows bred by intrauterine insemination. J. Swi. Heal. Prod., 12:182-185.

8. 8- Bolanos, I,M. ; Molina, J.R. \& Forsbrg, M. (1997). Effect of blood sampling \& administration of ACTH on cortisol \& progesterone levels in ovariectoized zebu cows. Acta. Vet. Scandinavica, 38:1-7.

9. 9-Gordon, I. (2003). Labarotory production of cattle embryos: The bovine oestrous cycle and associated events. Chapter2, pp.42-47. printed \& bound in the London.

10. 10-Stoebel, D.P. \& Mobery, G.P. (1982). Effect of adrenocorticotropin \& cortisol on luteinizing hormone surge \& estrous behavior in cows. J. Dairy Sci. , 65:1016-1024.

11. 11-Kawate, N. (2004). Studies on the regulation of expression of luteinizing hormone receptors in the ovary and the mechanisum of follicular cyst formation in ruminants. J. Reprod. Develop., Vol.50, (1):59.

12. 12-Vellucci, S.V. (1997). The autonomic and behavioural response to stress: Stress, stress hormones and the immune system.Ltd. Chichester, PP.49-70.

13. 13-Morales, R.J. ; Hernandez, C.J. \& Vazquez, G.J. (1998). Effect of hCG treatment at time of artificial insemination on the corpus luteum function \& the fertility of repeat-breeder Holstein cows. J. Vet. Mex. , 29:269-27.

14. 14-Nishigai, M. ; Kamomae, H. ; Tanaka, T. \& Kaneda, Y. (2002). Improvement of pregnancy rate in Japanese black cows by administration of hCG to recipients of transferred frozen-thawed embryos. Theriog., 58:1597-160..

15. 15-Bhattacharrya, H.K. and Hafiz, A. (2009). Treatment of delayed ovulation in dairy cattle. India. J. Ani. Res., Vol.43, Issue:3.

16. 16-Walton, J.S. ; Halbert, G.W. ; Robinson, N.A. \& Leslie, K.E. (1990). Effect of progesterone \& human chorionic gondotrophin administration five days postinsemination on plasma and milk concentration of progesterone \& pregnancy rates of normal \& repeat breeder dairy cows. J. Vet. Res., 54:305-308.

17. 17-Twardon, J. ; Dejneka, G.J. ; Blaszkowszowska, M. \& Trzesoski, P. (2002). The influence of simpanorm (carazolol) application on puerperal period course in dairy cows. Poland, Wroclaw, 59:216.

18. 18-Baggot, J.D. (2001). The physiological basis of veterianary clinical pharmacology. Blackwell Sci., Ltd. Printed \& Bound in reat British by MPG Book, chapter 4, Pp.140-143.

19. 19-Gustafsson, H. ; Larsson, K. ; Kindahl, H. \& Madej, A. (1986). Sequential endocrine changes \& behavior during oestrus and metaestrus in repeat breeder \& virgin heifers. Anim. Reprod. Sci. , 10:261-273.

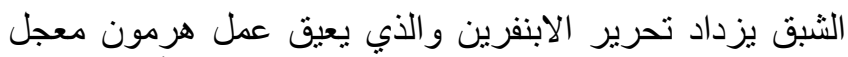

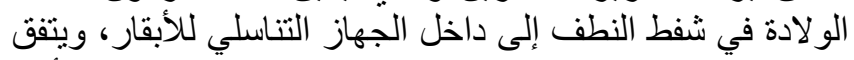

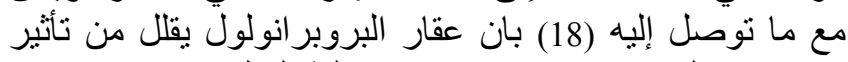
الابنفرين على مستقبلات بينا ب الادرينالية الفعل.

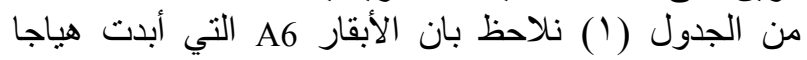

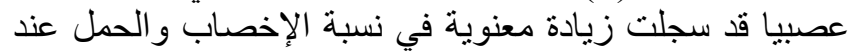

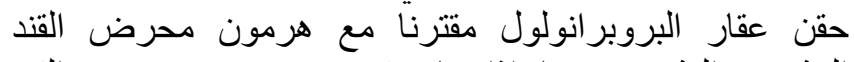

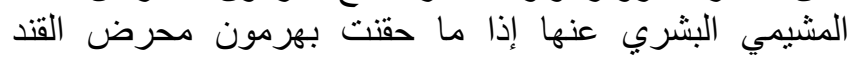

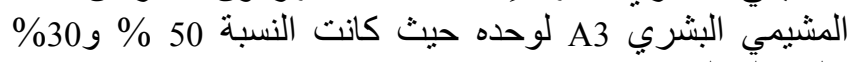

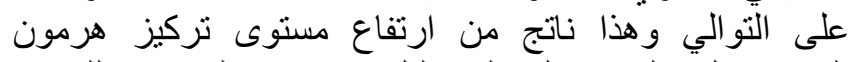

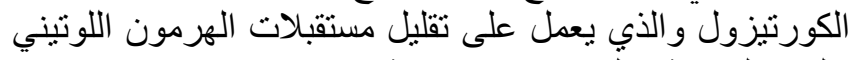

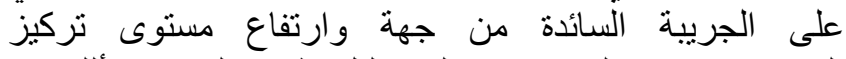

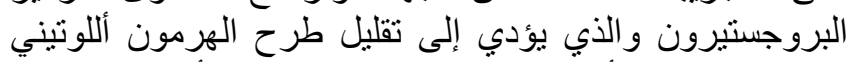

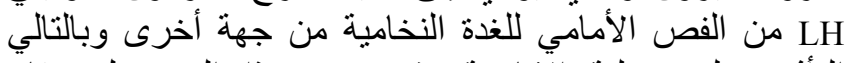

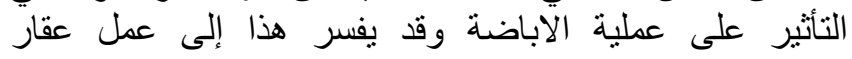

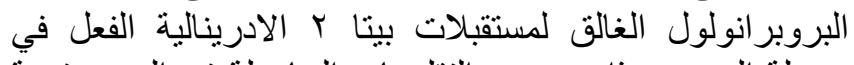

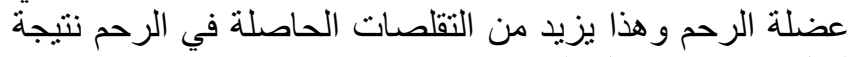

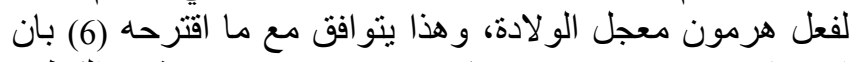

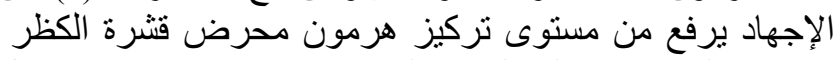

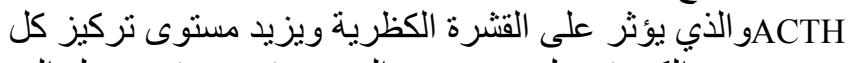

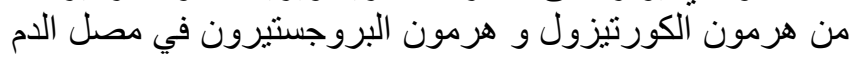

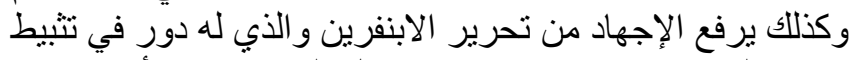

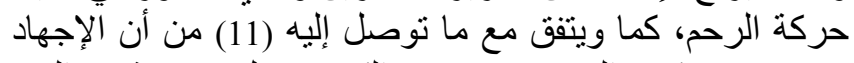

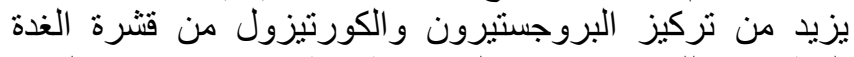

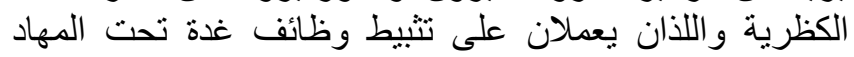
و الجريبة.

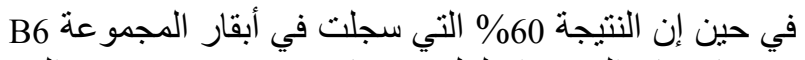

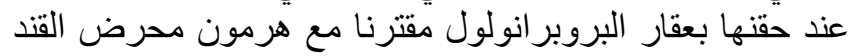

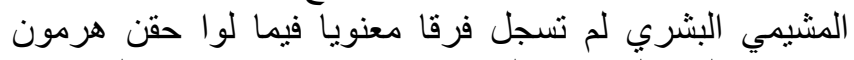

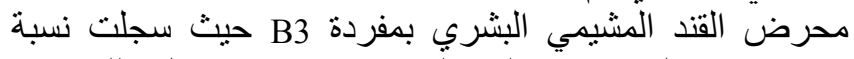

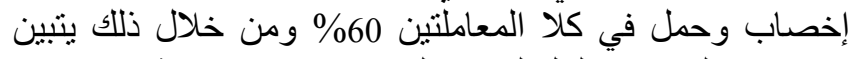

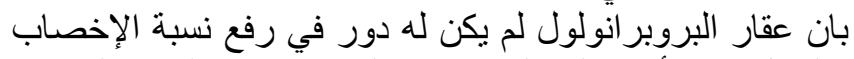

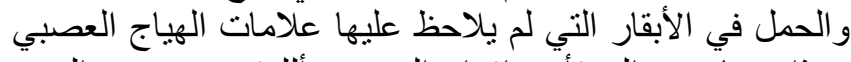

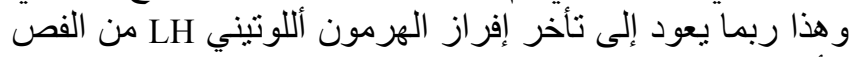

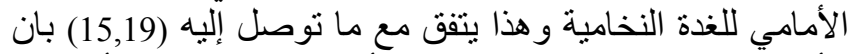

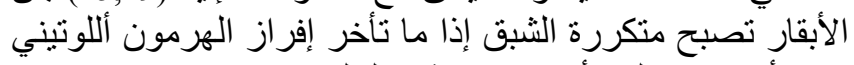

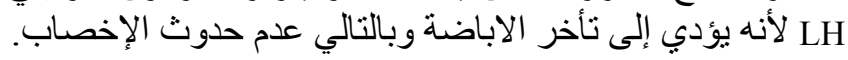

المصادر

1. 1-Bartlett, P.C. ; Kirk, J.H. \& Mather, E.C. (1986). Repeated insemination in Michigan Holstein - Friesian cattle: Incidence , 